\title{
Avaliação funcional e de sintomatologia dolorosa do pé e tornozelo em indivíduos com obesidade grave - Estudo controlado transversal*
}

\section{Functional Evaluation and Pain Symptomatology of the Foot and Ankle in Individuals with Severe Obesity - Controlled Transversal Study}

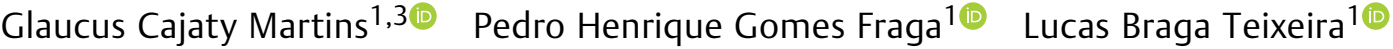 \\ Bruno Rodrigues Guterres Valle ${ }^{1 \odot}$ Luiz Felippe Martins Filho ${ }^{10}$ Maurício de Pinho Gama ${ }^{30}$
}

${ }^{1}$ Serviço de Ortopedia e Traumatologia, Hospital Federal de Ipanema,

Rio de Janeiro, RJ, Brasil

2 Serviço de Ortopedia e Traumatologia, Hospital Universitário da

Endereço para correspondência Glaucus Cajaty Martins, MD, PhD, Rua Jangadeiros, 67/69, Ipanema, Rio de Janeiro, RJ, 22420010, Brasil

Universidade Federal do Rio de Janeiro, RJ, Brasil

3 Serviço de Estatística Médica, Hospital Universitário da Universidade

Federal do Rio de Janeiro, RJ, Brasil

Rev Bras Ortop 2021;56(2):235-243.

\section{Resumo \\ Palavras-chave \\ - pé \\ - obesidade \\ - dor \\ - escala AOFAS}

Objetivo Avaliar a prevalência de queixas álgicas no pé e tornozelo, parâmetros radiográficos e o desempenho funcional de indivíduos com obesidade grave, Índice de Massa Corpórea (IMC) com valor $>40$ e indicação de cirurgia bariátrica.

Métodos Foram avaliados 40 pacientes com obesidade grave acompanhados em ambulatório de cirurgia bariátrica. Este grupo de obesos graves $(I M C>40)$ foi subdividido em dois subgrupos: obesos com IMC $<50(n=24)$; e outro de obesos com IMC > $50(n=16)$. Foi realizada comparação com grupo controle de 42 indivíduo voluntários com IMC médio de 24 . Foram avaliados a presença de dor no pé pela escala visual (EVA), o desempenho funcional pela escala da Associação Americana de Cirurgia do Pé e Tornozelo (AOFAS, na sigla em inglês) (domínios antepé, mediopé e retropé), idade, gênero, ângulo (âng) metatarso-falangeano do hálux, âng intermetatarsal do hálux , âng talocalcaneano, "pitch" calcaneano e âng de Meary.

Resultados Foi observada maior incidência de dor no pé no grupo de obesos graves em relação ao controle ( $p<0,0001$, razão de chances [odds ratio, OR]: 4,2). O

Trabalho desenvolvido no Hospital Federal de Ipanema, Rio de Janeiro, RJ, Brasil.

recebido

08 de Agosto de 2019

aceito

15 de Abril de 2020

Publicado online

Outubro 29, 2020
DOI https://doi.org/

$10.1055 / \mathrm{s}-0040-1713757$. ISSN 0102-3616. (c) 2020. Sociedade Brasileira de Ortopedia e Traumatologia. All rights reserved.

This is an open access article published by Thieme under the terms of the Creative Commons Attribution-NonDerivative-NonCommercial-License, permitting copying and reproduction so long as the original work is given appropriate credit. Contents may not be used for commercial purposes, or adapted, remixed, transformed or built upon. (https://creativecommons.org/ licenses/by-nc-nd/4.0/)

Thieme Revinter Publicações Ltda., Rua do Matoso 170, Rio de Janeiro, RJ, CEP 20270-135, Brazil 


\begin{abstract}
Keywords

- foot

- obesity

- pain

- AOFAS scale

Objective The present study aims to evaluate the prevalence of foot and ankle pain complaints, radiographic parameters, and functional performance in subjects with severe obesity (body mass index $[\mathrm{BMI}]>40$ ) who are candidates to bariatric surgery. Methods Forty severely obese patients were evaluated at a bariatric surgery outpatient facility. These severely obese subjects $(\mathrm{BMI}>40)$ were divided into two subgroups: those with $\mathrm{BMI}<50(n=24)$ and $\mathrm{BMI}>50(n=16)$. These patients were compared with a control group of 42 volunteers with a mean BMI value of 24 . The following parameters were assessed: foot pain (according to the visual analog scale [VAS]), functional performance (according to the American Orthopeadic Foot and Ankle Society [AOFAS] scale, including forefoot, midfoot and hindfoot domains), age, gender, hallux metatarsal-phalangeal angle, hallux intermetatarsal angle, talocalcaneal angle, calcanean pitch angle and Meary angle.

Results Incidence of foot pain was higher in the severely obese group compared with the control group ( $p<0.0001$; odds ratio [OR]: 4.2). Functional performance according to the AOFAS scale was lower in obese subjects compared with the control group $(p<0.0001$; OR for hindfoot, 4.81; OR for midfoot, 3.33).

Conclusion The incidence of foot pain was higher in the group of severely obese patients compared with the control group. According to the AOFAS scale, functional forefoot, midfoot and hindfoot performance was worse in severely obese individuals.
\end{abstract}

desempenho funcional pela escala AOFAS foi inferior no grupo de obesos em relação ao controle $(p<0,0001$, retropé com $O R=4,81$; mediopé com $O R=3,33)$.

Conclusão Houve maior incidência de dor no pé no grupo de obesos graves em relação ao controle. Houve pior desempenho funcional pela escala AOFAS nas regiões do antepé, mediopé e retropé no grupo de obesos graves.

\section{Introdução}

Atualmente, a obesidade é um dos problemas de saúde pública mais desafiadores da sociedade moderna. Estimase que haja atualmente no mundo mais de um bilhão de pessoas com sobrepeso, sendo que 300 milhões seriam consideradas obesas. ${ }^{1}$ Esta desordem acomete não apenas países desenvolvidos, mas também países em vias de desenvolvimento onde a ingestão de carboidratos, por serem alimentos baratos, é altamente difundida. ${ }^{2}$

Estudos populacionais demonstraram que a obesidade é fator de risco independente para a manifestação de dor e artrose de joelho. ${ }^{3-5}$

A prevalência de dor comprometendo os pés de indivíduos obesos foi menos estudada na literatura do que as alterações dolorosas em joelhos relacionadas à obesidade. ${ }^{3-5}$

A obesidade ocasionaria dor e alterações biomecânicas no pé secundariamente à deformidade em pé plano, alterações na gordura plantar, diminuição da força muscular e modificações no padrão da marcha. ${ }^{6}$ Este processo geraria perda de equilíbrio, risco de queda e interferiria na mobilidade destes indivíduos. ${ }^{6,7}$

Atualmente, há uma nova vertente de estudos com a descoberta de proteínas geradas a partir da gordura visceral: as adipocinas. A mais conhecida seria a leptina, que tem papéis na ação da insulina e na produção de citocinas inflamatórias nos condrócitos. ${ }^{8}$ Estudos recentes demonstraram maior incidência de dor e artrose em joelhos relacionadas a níveis elevados de adipocinas séricas e à síndrome metabólica. ${ }^{9}$

Em indivíduos obesos graves com índice de massa corpórea $(\mathrm{IMC})>40$, a prevalência de dor, o nível de desempenho funcional e os parâmetros radiográficos de alinhamento no que se refere ao pé e ao tornozelo ainda não foram suficientemente descritos na literatura. A compilação destes dados se faz necessária para o adequado dimensionamento da importância do problema. A determinação de parâmetros clínicos e de imagem que possam indicar a necessidade de intervenção precoce tem papel fundamental na formulação de estratégias terapêuticas e preventivas neste grupo crescente de pessoas normalmente em idade economicamente ativa.

$O$ presente estudo tem como objetivo principal avaliar a prevalência de dor medida pela escala visual analógica (EVA) em uma amostra de indivíduos obesos graves (IMC > 40) em relação a um grupo controle de indivíduos com IMC médio de 24. Como objetivos secundários, serão avaliadas as diferenças funcionais medidas pela escala AOFAS ${ }^{10}$ e parâmetros radiográficos para a avaliação morfológica do pé nos grupos estudados. Como hipótese inicial, seria esperada maior prevalência de dor e desempenho funcional inferior nos indivíduos com obesidade grave em relação ao grupo controle. 
Tabela 1 Grupo de estudo(obesos)

\begin{tabular}{|c|c|c|c|c|c|c|c|c|c|c|c|c|c|c|c|}
\hline & SEXO & IDADE & PESO & CLASSIFICAÇÃO & ALTURA & IMC & VAS & LADO & $A R$ & A M & A A & Âng AMF $^{\circ}$ & Âng $\mathrm{AIM}^{\circ}$ & Âng APC & Âng ATC \\
\hline 1 & $\mathrm{~F}$ & 61 & 92 & PAC & 1,5 & 40,9 & 9 & ESQ & 47 & 79 & 93 & normal & normal & NORMAL & PLANO \\
\hline 2 & $\mathrm{~F}$ & 42 & 110 & PAC & 1,64 & 40,9 & 9 & DIR & 49 & 60 & 95 & leve & normal & NORMAL & NORMAL \\
\hline 3 & $\mathrm{~F}$ & 64 & 105 & PAC & 1,6 & 41 & 8 & DIR & 28 & 52 & 100 & normal & normal & NORMAL & PLANO \\
\hline 4 & $\mathrm{~F}$ & 45 & 108 & PAC & 1,62 & 41,2 & 10 & ESQ & 65 & 53 & 74 & normal & leve & NORMAL & NORMAL \\
\hline 5 & $\mathrm{~F}$ & 32 & 99 & PAC & 1,55 & 41,6 & 8 & DIR & 50 & 65 & 93 & normal & moderado & PLANO & NORMAL \\
\hline 6 & M & 27 & 158 & PAC & 1,95 & 41,6 & 8 & ESQ & 85 & 82 & 85 & normal & normal & NORMAL & NORMAL \\
\hline 7 & $\mathrm{~F}$ & 47 & 97,5 & PAC & 152 & 42,2 & 7 & DIR & 67 & 64 & 95 & normal & normal & NORMAL & NORMAL \\
\hline 8 & $M$ & 47 & 123 & PAC & 1,7 & 42,6 & 0 & ESQ & 80 & 85 & 85 & normal & normal & NORMAL & NORMAL \\
\hline 9 & $M$ & 62 & 108 & PAC & 1,59 & 42,7 & 8 & DIR & 72 & 76 & 93 & normal & moderado & NORMAL & NORMAL \\
\hline 10 & M & 25 & 143 & PAC & 1,83 & 42,7 & 10 & DIR & 67 & 100 & 100 & moderado & moderado & NORMAL & NORMAL \\
\hline 11 & M & 30 & 128 & PAC & 1,73 & 42,77 & 7 & DIR & 82 & 100 & 73 & normal & leve & NORMAL & NORMAL \\
\hline 12 & $M$ & 40 & 150 & PAC & 1,87 & 42,9 & 6 & DIR & 79 & 85 & 82 & normal & normal & NORMAL & PLANO \\
\hline 13 & $\mathrm{~F}$ & 58 & 136 & PAC & 1,77 & 43,4 & 9 & DIR & 30 & 32 & 83 & moderado & grave & NORMAL & NORMAL \\
\hline 14 & $\mathrm{~F}$ & 53 & 102 & PAC & 1,52 & 44,15 & 5 & DIR & 67 & 100 & 100 & moderado & moderado & NORMAL & NORMAL \\
\hline 15 & $\mathrm{~F}$ & 19 & 109 & PAC & 1,57 & 44,2 & 8 & DIR & 67 & 49 & 100 & moderado & leve & NORMAL & NORMAL \\
\hline 16 & $\mathrm{~F}$ & 38 & 122 & PAC & 1,66 & 44,3 & 9 & ESQ & 80 & 82 & 93 & normal & leve & PLANO & NORMAL \\
\hline 17 & $\mathrm{~F}$ & 49 & 103 & PAC & 1,52 & 44,58 & 8 & DIR & 71 & 56 & 100 & normal & normal & NORMAL & NORMAL \\
\hline 18 & $\mathrm{~F}$ & 47 & 124 & PAC & 1,65 & 45,5 & 7 & DIR & 72 & 60 & 77 & normal & moderado & NORMAL & CAVO \\
\hline 19 & $\mathrm{~F}$ & 53 & 97 & PAC & 1,52 & 45,8 & 0 & DIR & 88 & 89 & 90 & normal & leve & NORMAL & NORMAL \\
\hline 20 & $\mathrm{~F}$ & 43 & 126 & PAC & 1,65 & 46,3 & 8 & DIR & 68 & 45 & 90 & normal & normal & NORMAL & CAVO \\
\hline 21 & $\mathrm{~F}$ & 63 & 116 & PAC & 1,58 & 46,5 & 7 & DIR & 52 & 87 & 100 & normal & leve & CAVO & NORMAL \\
\hline 22 & M & 50 & 142 & PAC & 1,72 & 48 & 6 & DIR & 60 & 100 & 78 & moderado & moderado & NORMAL & NORMAL \\
\hline 23 & $\mathrm{~F}$ & 63 & 115 & PAC & 1,55 & 48,87 & 8 & ESQ & 82 & 100 & 73 & moderado & leve & PLANO & NORMAL \\
\hline 24 & $\mathrm{~F}$ & 33 & 140 & PAC & 1,68 & 49,6 & 5 & DIR & 67 & 89 & 70 & leve & moderado & NORMAL & NORMAL \\
\hline 25 & $\mathrm{~F}$ & 52 & 130 & PAC & 1,61 & 50,2 & 3 & ESQ & 78 & 75 & 100 & moderado & leve & NORMAL & NORMAL \\
\hline 26 & $\mathrm{M}$ & 61 & 146 & PAC & 1,69 & 51,12 & 10 & ESQ & 57 & 100 & 57 & leve & leve & NORMAL & PLANO \\
\hline 27 & $\mathrm{~F}$ & 45 & 120 & PAC & 1,53 & 51,26 & 3 & DIR & 73 & 62 & 100 & moderado & moderado & NORMAL & NORMAL \\
\hline 28 & $\mathrm{~F}$ & 41 & 140 & PAC & 1,65 & 51,42 & 8 & DIR & 93 & 53 & 100 & moderado & leve & NORMAL & NORMAL \\
\hline 29 & $\mathrm{~F}$ & 44 & 120 & PAC & 1,52 & 51,94 & 5 & ESQ & 84 & 82 & 100 & leve & leve & PLANO & NORMAL \\
\hline 30 & $\mathrm{~F}$ & 55 & 116 & PAC & 1,48 & 52,16 & 8 & ESQ & 51 & 100 & 100 & leve & moderado & NORMAL & NORMAL \\
\hline 31 & $\mathrm{~F}$ & 42 & 113 & PAC & 1,47 & 52,29 & 6 & DIR & 50 & 45 & 72 & normal & normal & NORMAL & CAVO \\
\hline 32 & $\mathrm{~F}$ & 36 & 123 & PAC & 1,53 & 52,59 & 5 & ESQ & 58 & 100 & 100 & moderado & moderado & NORMAL & NORMAL \\
\hline 33 & $\mathrm{~F}$ & 27 & 135 & PAC & 1,58 & 54,08 & 7 & DIR & 41 & 61 & 100 & normal & normal & NORMAL & NORMAL \\
\hline 34 & $\mathrm{~F}$ & 37 & 145 & PAC & 1,62 & 55,25 & 7 & ESQ & 64 & 100 & 100 & leve & leve & NORMAL & NORMAL \\
\hline 35 & $\mathrm{~F}$ & 51 & 133 & PAC & 1,55 & 55,4 & 10 & ESQ & 29 & 39 & 90 & normal & normal & NORMAL & NORMAL \\
\hline 36 & $\mathrm{~F}$ & 40 & 132 & PAC & 1,54 & 55,66 & 8 & DIR & 81 & 100 & 83 & moderado & moderado & NORMAL & NORMAL \\
\hline 37 & $\mathrm{M}$ & 40 & 165 & PAC & 1,72 & 55,77 & 5 & ESQ & 69 & 100 & 70 & moderado & leve & NORMAL & NORMAL \\
\hline 38 & $\mathrm{~F}$ & 36 & 162 & PAC & 1,65 & 59,5 & 7 & ESQ & 88 & 73 & 95 & normal & leve & NORMAL & NORMAL \\
\hline 39 & $\mathrm{~F}$ & 37 & 153 & PAC & 1,58 & 61,29 & 5 & DIR & 55 & 64 & 100 & normal & normal & NORMAL & NORMAL \\
\hline 40 & M & 59 & 181 & PAC & 1,79 & 71,6 & 7 & DIR & 89 & 100 & 100 & normal & grave & NORMAL & NORMAL \\
\hline
\end{tabular}

Abreviações: AR, abreviatura da escala AOFAS do retropé; AM, abreviatura da escala AOFAS do mediopé; AA, abreviatura da escala AOFAS do antepé; Âng AIM, ângulo intermetatarsal; Âng AMF, ângulo metatarso falangeano do hálux; Âng APC, ângulo "pitch" calcaneano; Âng ATC, ângulo talocalcaneano; DIR, direito; ESQ, esquerdo; IMC, índice de massa corporal; PAC, paciente; VAS, escala visual analógica.

\section{Metodologia}

O presente estudo está de acordo com as normas da convenção de Helsinque e foi aprovado pela comissão de ética em pesquisa do hospital (CAAE: 69073215.2.0000.5646, parecer 2.127.775). Todos os participantes (obesos e grupo controle) assinaram termo de consentimento informado.

No presente trabalho, o estudo foi do tipo observacional transversal. Os dados da presente pesquisa foram coletados pelos residentes de ortopedia do serviço no período de junho de 2017 a abril de 2018.

Foram incluídos 40 pacientes (-Tabela 1 ) oriundos do ambulatório de cirurgia bariátrica de nosso hospital. A casuística consistiu de 10 homens e 30 mulheres, com idade média de 45,45 anos (25 a 63 anos).

Procurou-se estruturar um grupo controle pareado por sexo e mesma faixa etária de indivíduos voluntários (funcionários, residentes e pacientes do 
Tabela 2 Grupo controle

\begin{tabular}{|c|c|c|c|c|c|c|c|c|c|c|c|c|c|c|c|c|}
\hline & SEXO & IDADE & PESO & CLASSIFICAÇÃO & ALTURA & IMC & VAS & LADO & A R & A M & A A & $\begin{array}{l}\text { Ang } \\
\text { AMF }^{\circ}\end{array}$ & $\begin{array}{l}\text { Ang } \\
\text { AlM }^{\circ}\end{array}$ & $\begin{array}{l}\text { Ang } \\
\text { APC }^{\circ}\end{array}$ & Ang ATC & $\begin{array}{l}\text { Ang } \\
\text { AM }\end{array}$ \\
\hline 1 & $\mathrm{~F}$ & 61 & 80 & CONTROLE & 1,62 & 30,48 & 3 & ESQ & 49 & 66 & 100 & normal & normal & CAVO & NORMAL & CAVO \\
\hline 2 & $\mathrm{~F}$ & 58 & 61 & CONTROLE & 1,54 & 25,72 & 3 & ESQ & 41 & 58 & 100 & moderado & leve & NORMAL & NORMAL & NORMAL \\
\hline 3 & $\mathrm{~F}$ & 45 & 62 & CONTROLE & 1,54 & 26,14 & 0 & DIR & 100 & 100 & 100 & normal & leve & NORMAL & NORMAL & NORMAL \\
\hline 4 & $\mathrm{~F}$ & 44 & 65 & CONTROLE & 1,68 & 23,03 & 0 & DIR & 58 & 58 & 100 & normal & moderado & PLANO & NORMAL & PLANO \\
\hline 5 & $\mathrm{~F}$ & 38 & 74 & CONTROLE & 1,74 & 22,4 & 0 & ESQ & 60 & 90 & 90 & normal & normal & NORMAL & NORMAL & NORMAL \\
\hline 6 & $\mathrm{~F}$ & 54 & 89 & CONTROLE & 1,7 & 30,45 & 3 & DIR & 41 & 56 & 100 & normal & moderado & PLANO & NORMAL & PLANO \\
\hline 7 & $\mathrm{~F}$ & 27 & 57 & CONTROLE & 1,71 & 19,15 & 0 & DIR & 100 & 100 & 100 & normal & normal & NORMAL & NORMAL & NORMAL \\
\hline 8 & $\mathrm{~F}$ & 38 & 59 & CONTROLE & 1,63 & 22,21 & 5 & ESQ & 100 & 100 & 100 & moderado & leve & NORMAL & NORMAL & NORMAL \\
\hline 9 & $\mathrm{~F}$ & 60 & 62 & CONTROLE & 1,59 & 24,52 & 0 & DIR & 57 & 53 & 100 & leve & leve & NORMAL & NORMAL & NORMAL \\
\hline 10 & $\mathrm{~F}$ & 35 & 69 & CONTROLE & 1,71 & 23,6 & 0 & DIR & 100 & 100 & 100 & leve & leve & NORMAL & NORMAL & NORMAL \\
\hline 11 & $\mathrm{~F}$ & 37 & 72 & CONTROLE & 1,73 & 24,06 & 6 & ESQ & 100 & 100 & 100 & leve & leve & PLANO & NORMAL & PLANO \\
\hline 12 & $\mathrm{~F}$ & 53 & 69 & CONTROLE & 1,62 & 26,29 & 7 & DIR & 58 & 66 & 100 & moderado & moderado & PLANO & NORMAL & PLANO \\
\hline 13 & $\mathrm{~F}$ & 41 & 72 & CONTROLE & 1,7 & 24,91 & 4 & ESQ & 100 & 100 & 100 & leve & leve & NORMAL & NORMAL & NORMAL \\
\hline 14 & $\mathrm{~F}$ & 20 & 54 & CONTROLE & 1,59 & 21,36 & 0 & DIR & 100 & 100 & 100 & normal & normal & NORMAL & NORMAL & NORMAL \\
\hline 15 & $\mathrm{M}$ & 42 & 87 & CONTROLE & 1,81 & 26,56 & 0 & DIR & 100 & 100 & 100 & normal & normal & NORMAL & NORMAL & NORMAL \\
\hline 16 & M & 30 & 70 & CONTROLE & 1,71 & 23,66 & 0 & DIR & 100 & 100 & 100 & moderado & moderado & NORMAL & NORMAL & NORMAL \\
\hline 17 & M & 47 & 74 & CONTROLE & 1,73 & 25,01 & 4 & ESQ & 100 & 100 & 100 & normal & leve & NORMAL & NORMAL & NORMAL \\
\hline 18 & $M$ & 24 & 76 & CONTROLE & 1,74 & 25,1 & 0 & DIR & 100 & 100 & 100 & moderado & normal & NORMAL & NORMAL & NORMAL \\
\hline 19 & $\mathrm{M}$ & 27 & 78 & CONTROLE & 1,7 & 26,99 & 0 & DIR & 100 & 100 & 100 & normal & normal & NORMAL & NORMAL & NORMAL \\
\hline 20 & $M$ & 35 & 76 & CONTROLE & 1,69 & 26,61 & 6 & ESQ & 100 & 100 & 100 & moderado & leve & NORMAL & NORMAL & NORMAL \\
\hline 21 & $\mathrm{M}$ & 61 & 76 & CONTROLE & 1,72 & 25,69 & 0 & DIR & 53 & 49 & 100 & normal & normal & NORMAL & NORMAL & NORMAL \\
\hline 22 & $\mathrm{M}$ & 56 & 92 & CONTROLE & 1,79 & 28,71 & 3 & DIR & 100 & 100 & 100 & leve & leve & NORMAL & NORMAL & NORMAL \\
\hline 23 & $\mathrm{~F}$ & 25 & 50 & CONTROLE & 1,65 & 18,4 & 0 & ESQ & 100 & 100 & 100 & normal & leve & NORMAL & NORMAL & NORMAL \\
\hline 24 & $\mathrm{~F}$ & 56 & 52 & CONTROLE & 1,6 & 20,3 & 0 & DIR & 100 & 100 & 100 & normal & normal & NORMAL & NORMAL & CAVO \\
\hline 25 & $\mathrm{~F}$ & 35 & 55 & CONTROLE & 1,53 & 23,5 & 0 & DIR & 100 & 100 & 75 & moderado & normal & NORMAL & NORMAL & NORMAL \\
\hline 26 & $\mathrm{~F}$ & 50 & 57 & CONTROLE & 1,6 & 22,3 & 3 & DIR & 100 & 90 & 100 & normal & normal & NORMAL & NORMAL & CAVO \\
\hline 27 & $\mathrm{~F}$ & 33 & 65 & CONTROLE & 1,68 & 23 & 1 & DIR & 100 & 100 & 92 & normal & leve & NORMAL & NORMAL & NORMAL \\
\hline 28 & M & 34 & 79 & CONTROLE & 1,81 & 24 & 0 & DIR & 100 & 100 & 100 & normal & leve & NORMAL & NORMAL & CAVO \\
\hline 29 & $\mathrm{~F}$ & 60 & 60 & CONTROLE & 1,54 & 25,3 & 2 & ESQ & 88 & 100 & 100 & normal & leve & NORMAL & NORMAL & NORMAL \\
\hline 30 & $\mathrm{~F}$ & 47 & 575 & CONTROLE & 1,67 & 20,4 & 0 & DIR & 100 & 100 & 100 & normal & normal & NORMAL & NORMAL & NORMAL \\
\hline 31 & $\mathrm{~F}$ & 61 & 57 & CONTROLE & 1,53 & 24,3 & 0 & DIR & 100 & 100 & 100 & normal & normal & NORMAL & NORMAL & NORMAL \\
\hline 32 & $\mathrm{~F}$ & 51 & 54 & CONTROLE & 1,55 & 22,5 & 0 & ESQ & 100 & 100 & 100 & normal & normal & CAVO & NORMAL & CAVO \\
\hline 33 & M & 58 & 73 & CONTROLE & 1,7 & 25,3 & 0 & DIR & 100 & 100 & 100 & normal & normal & NORMAL & NORMAL & NORMAL \\
\hline 34 & $\mathrm{M}$ & 52 & 83 & CONTROLE & 1,8 & 25 & 0 & ESQ & 100 & 100 & 100 & normal & normal & NORMAL & NORMAL & CAVO \\
\hline 35 & $\mathrm{~F}$ & 40 & 62 & CONTROLE & 1,71 & 21,2 & 0 & ESQ & 100 & 100 & 100 & normal & leve & NORMAL & NORMAL & NORMAL \\
\hline 36 & $\mathrm{~F}$ & 42 & 58 & CONTROLE & 1,55 & 24,1 & 5 & ESQ & 90 & 100 & 100 & normal & normal & NORMAL & NORMAL & NORMAL \\
\hline 37 & $\mathrm{~F}$ & 53 & 60 & CONTROLE & 1,57 & 24,3 & 2 & DIR & 87 & 92 & 92 & moderado & moderado & NORMAL & PLANO & NORMAL \\
\hline 38 & $\mathrm{~F}$ & 60 & 49 & CONTROLE & 1,55 & 20,4 & 6 & ESQ & 64 & 95 & 100 & normal & normal & NORMAL & NORMAL & NORMAL \\
\hline 39 & $\mathrm{M}$ & 27 & 82 & CONTROLE & 1,79 & 25,2 & 0 & DIR & 100 & 100 & 100 & normal & moderado & NORMAL & NORMAL & NORMAL \\
\hline 40 & $\mathrm{~F}$ & 43 & 54 & CONTROLE & 1,53 & 23,1 & 0 & ESQ & 85 & 85 & 75 & normal & moderado & NORMAL & NORMAL & NORMAL \\
\hline 41 & $\mathrm{~F}$ & 48 & 63 & CONTROLE & 1,59 & 24,9 & 0 & ESQ & 75 & 72 & 75 & moderado & grave & NORMAL & NORMAL & NORMAL \\
\hline 42 & $\mathrm{~F}$ & 35 & 75 & CONTROLE & 1,75 & 24,5 & 0 & ESQ & 100 & 100 & 100 & normal & leve & NORMAL & NORMAL & NORMAL \\
\hline
\end{tabular}

Abreviações: AR, abreviatura da escala AOFAS do retropé; AM, abreviatura da escala AOFAS do mediopé; AA, abreviatura da escala AOFAS do antepé; Âng AIM, ângulo intermetatarsal; Âng AMF, ângulo metatarso falangeano do hálux; Âng APC, ângulo "pitch" calcaneano; Âng ATC, ângulo talocalcaneano; DIR, direito; ESQ, esquerdo; IMC, índice de massa corporal; VAS, escala visual analógica.

ambulatório de Cirurgia Geral não bariátrica) para comparação com o grupo de indivíduos obesos (-Tabela 2). 0 grupo controle consistiu de 42 indivíduos, sendo 12 homens e 30 mulheres com idade média de 43,9 anos (24 a 61 anos).

Como critérios de inclusão no grupo de estudo, foram aceitos indivíduos com IMC $>40$, indicação de cirurgia bariátrica, $>18$ anos e que consentiram em participar do estudo.
Como critérios de exclusão, foram descartados da pesquisa indivíduos previamente já submetidos a procedimentos cirúrgicos de origem ortopédica, vascular ou dermatológica e plástica em qualquer segmento dos membros inferiores (quadril, joelho, tornozelo e pé). Indivíduos com sequelas de fraturas em membros inferiores ou patologias com indicação cirúrgica já estabelecida em membros inferiores, seja ortopédica (artrose de quadril ou joelho, artrose de tornozelo ou 
pé) ou vascular (insuficiência arterial ou venosa, úlceras, necrose digital ou cutânea) foram excluídos da pesquisa.

Foram aferidos no Ambulatório de Cirurgia Bariátrica o peso e a altura. O IMC foi calculado pela divisão do peso em $\mathrm{Kg}$ pelo quadrado da altura em metros. A fim de classificação: IMC $<20$ (subpeso), 20 a 24,9 (normal), 25 a 29,9 (sobrepeso) e $>30$ (obesidade), $>40$ (obesidade mórbida) e $>50$ (superobeso). ${ }^{7}$

Foram realizadas radiografias com carga em anteposterior (AP) e perfil com carga de ambos os pés dos participantes na pesquisa (obesos e grupo controle).

Os parâmetros radiológicos aferidos foram:

1) ângulo (âng) metatarso-falangeano do hálux (AMF) medido no AP - normal $<15^{\circ}$; leve 15 a $19^{\circ}$; moderado $\left(20\right.$ a $\left.39^{\circ}\right)$; grave $\left(>40^{\circ}\right)$;

2) ângulo intermetatarsal (AIM) medido no AP - normal < 9; leve (9-11); moderado (12-15); grave > 16;

3) ângulo talocalcaneano (ATC) medido no AP cavo $<20^{\circ}$; normal (entre 20 e $30^{\circ}$ ); plano $>40^{\circ}$;

4) ângulo "pitch" calcaneano (APC) medido no perfil plano $<10$; normal (10-30); cavo $>30$;

5) ângulo Meary (AM) ou âng talus-primeiro metatarsiano medido no perfil. Valor normal seria zero. Acima de $10^{\circ}$ de desvio plantar indicaria pé cavo; e desvio dorsal $>10^{\circ}$ indicaria pé plano.

Os ângs AMF e AIM avaliam e graduam a deformidade em hálux valgo. Os ângs ATC, APC e AM avaliam se o pé se apresenta dentro da normalidade ou demonstra deformidades em pé plano ou cavo.

A dor nas atividades da vida diária (deambulação, subir e descer escadas, repouso) foi avaliada de forma simples pela escala de dor visual analógica (EVA), variando de 0 (sem dor) a 10 (dor mais intensa possível). Com a finalidade de avaliação mais objetiva, a dor classificada de 1 a 3 foi considerada leve, de 4 a 6 moderada, e de 7 a 10 forte. Valores $>3$ foram anotados como significativos para razão de chances (odds ratio [OR]).

A escala da Associação Americana de Cirurgia do Pé e Tornozelo (AOFAS, na sigla em inglês) ${ }^{10}$ foi utilizada para avaliação funcional dos pés. Esta classificação aborda parâmetros de dor, função, uso de calçados, distância percorrida, mal alinhamento do pé e padrão de marcha. Esta escala analisa separadamente o antepé (hálux; pequenos dedos), o mediopé e o retropé/tornozelo. Esta escala em cada um de seus 3 domínios apresenta valores decrescentes de 100 até 0 (valor $<70$ seria resultado insatisfatório).

Foram correlacionados estatisticamente entre os grupos de estudo e controle os parâmetros: EVA, escala AOFAS (A: antepé, M: mediopé e R: retropé), ângs AMF, AM, ATC, APC e AIM. As relações entre EVA e idade e EVA e sexo também foram avaliadas nos grupos estudados.

Foi realizada a correlação entre o EVA e IMC.

No grupo de obesos, foram constituídos dois subgrupos: obesidade mórbida com IMC entre 40 e 50 e obesidade supermórbida com IMC > 50, com 24 e 16 membros respectivamente. Os parâmetros VAS e escala AOFAS foram avaliados comparativamente entre estes dois subgrupos.

No presente estudo, a análise dos dados foi realizada focando-se no membro mais sintomático (maior responsável pela EVA). Tal metodologia visa evitar comprometimento da análise estatística caso ambos membros (pés) sejam avaliados como unidades estatísticas separadas conforme previamente descrito por Menz. ${ }^{11}$

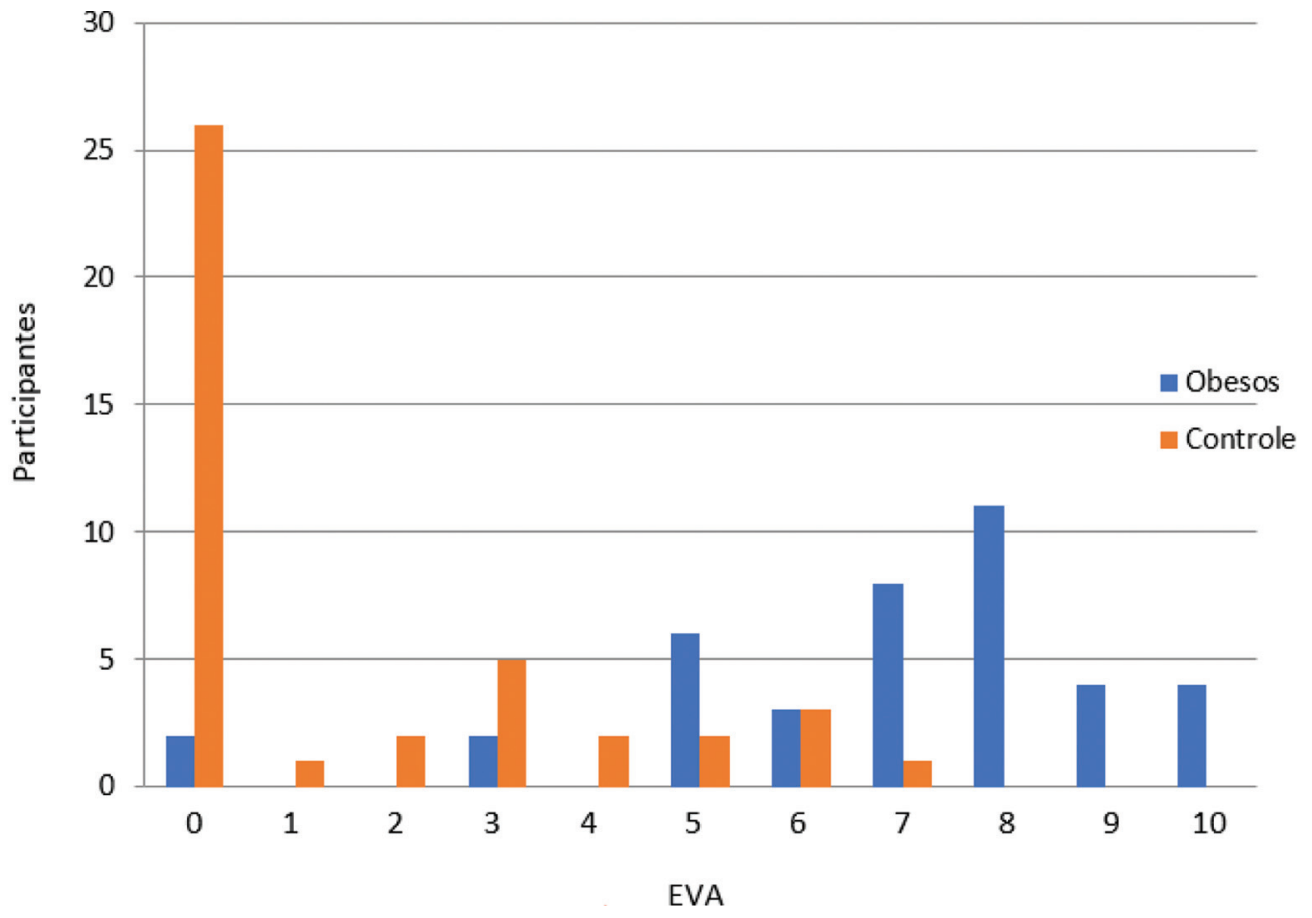

Fig. 1 Gráfico 2D escala de dor (VAS) no grupo de estudo de obesos (azul) e controle (vermelho). 
Tabela 3 Resultados da correlação entre o grupo obeso e controle

\begin{tabular}{|c|c|c|c|}
\hline PARÂMETRO & GRUPO OBESO & GRUPO CONTROLE & ESTATÍSTICA \\
\hline Gênero & M 25\%/ F 75\% & M 28,5\% / F 71,5\% & $p=0,539$ \\
\hline Idade & $44,85 \%$ & 43,8 (Média) & $p=0,3554$ \\
\hline VAS & $95 \%$ com dor & $40 \%$ com dor & $p=0,0001$ \\
\hline $\begin{array}{l}\text { AOFAS R } \\
\text { (valor médio) }\end{array}$ & 65.87 & 88.23 & $p<0,0001$ \\
\hline $\begin{array}{l}\text { AOFAS M } \\
\text { (valor médio) }\end{array}$ & 76,1 & 91,19 & $p<0,0001$ \\
\hline $\begin{array}{l}\text { AOFAS A } \\
\text { (valor médio) }\end{array}$ & 89,59 & 97,59 & $p<0,0001$ \\
\hline \multirow[t]{3}{*}{ angAPC } & $\mathrm{N}=87,5 \%$ & $\mathrm{~N}=85,71 \%$ & \multirow[t]{3}{*}{$p=0,8585$} \\
\hline & $C=2,5 \%$ & $C=4,70 \%$ & \\
\hline & $P=10 \%$ & $P=9,52 \%$ & \\
\hline \multirow[t]{3}{*}{ angATC } & $\mathrm{N}=82,5 \%$ & $\mathrm{~N}=97,6 \%$ & \multirow[t]{3}{*}{$p=0,031$} \\
\hline & $C=7,5 \%$ & $C=0 \%$ & \\
\hline & $\mathrm{P}=10 \%$ & $\mathrm{P}=2,6 \%$ & \\
\hline \multirow[t]{3}{*}{ angAM } & $\mathrm{N}=67,5 \%$ & $N=76,2 \%$ & \multirow[t]{3}{*}{$p=0,743$} \\
\hline & $C=12,5 \%$ & $C=14,3 \%$ & \\
\hline & $P=17,5 \%$ & $P=9,5 \%$ & \\
\hline angAMF & $\mathrm{N}=45 \%$ & $\mathrm{~N}=66.6 \%$ & $p=0,743$ \\
\hline angAIM & $N=30 \%$ & $\mathrm{~N}=42,85 \%$ & $p=0,54$ \\
\hline \multirow[t]{2}{*}{ Gênero x VAS } & \multirow[t]{2}{*}{$-\ldots$} & \multirow[t]{2}{*}{$-\ldots+\ldots-\ldots$} & $p=0,33$ obeso \\
\hline & & & $p=0,6417$ controle \\
\hline \multirow[t]{2}{*}{ IMC X VAS } & \multirow[t]{2}{*}{-} & \multirow[t]{2}{*}{ - - _ - } & $p=0,1407$ obeso \\
\hline & & & $p=0,2343$ controle \\
\hline
\end{tabular}

Abreviações: AOFAS R, Escala funcional da Associação Americana de Cirurgia do Pé e Tornozelo relativa ao retropé; AOFAS M, Escala funcional da Associação Americana de Cirurgia do Pé e Tornozelo relativa ao mediopé; AOFAS A, Escala funcional da Associação Americana de Cirurgia do Pé e Tornozelo relativa ao antepé; Âng AIM, ângulo intermetatarsal; Âng AMF, ângulo metatarso falangeano do hálux; Âng APC, ângulo "pitch" calcaneano; Âng ATC, ângulo talocalcaneano; C, cavo; F, feminino; M, masculino; N, normal; $p=$ Estatística; VAS, escala visual analógica.

\section{Análise Estatística}

Foi utilizado o teste G2 Wilks para avaliar o parâmetro dor (EVA) entre os grupos controle e obesos, entre os subgrupos de obesos e para avaliar a relação entre EVA e gênero e EVA e idade. Este teste também foi utilizado para análise dos ângs radiográficos (AMF, AIM, APC, AM e ATC). O teste de MannWhitney foi utilizado para análise da escala AOFAS. Utilizouse a matriz de correlação de Pearson para correlação entre EVA e IMC. Foi determinado como significativo $p<0,05$.

\section{Resultados}

A dor nos pés foi referida por 38/40 (95\%) pacientes obesos e por 16/42 (38\%) controles (-Figura 1). Nos obesos, houve 19 $(47,5 \%)$ casos de dor grave, 17 (42,5\%) de dor moderada, 2 (5\%) de dor leve e 2 (5\%) sem dor. No grupo controle, houve 8 $(19,05 \%)$ casos de dor moderada, 8 (19,05\%) de dor leve e 26 $(61,90 \%)$ sem dor.
Não houve diferença entre os grupos de obesos e controle em relação aos parâmetros idade e gênero, testes t de student $(p=0,3554)$ e teste de diferença de proporções $(p=0,539)$, respectivamente ( $\mathbf{- T a b e l a} \mathbf{3}$ ).

A escala de dor referida (EVA) mostrou maior prevalência de dor nos pés do grupo obesos em relação ao controle pelo teste de G2-Wilks ( $p=0,0001$ ); OR: 4,2.

A escala AOFAS em seus três domínios: antepé, mediopé e retropé, mostrou desempenho funcional inferior no grupo obeso em relação ao controle, teste de Mann-Whitney $(p<0,0001)$ (-Figura 2). Retropé com OR $=4,810$. Mediopé com $\mathrm{OR}=3,33$. Antepé: não foi possível calcular o $\mathrm{OR}$ pois nenhum individuo no controle teve valor $<70$.

A dor avaliada pela escala EVA não teve relação com o IMC no grupo de estudo ou no controle, respectivamente, testes de Pearson $(p=-0,1407)$ e $(p=0,2343)$.

O ângulo ATC evidenciou valores mais elevados no grupo de obesos, configurando maior prevalência de pés com padrão plano, teste G2 Wilks ( $p=0,0317)$. 
AOFAS ANTEPÉ

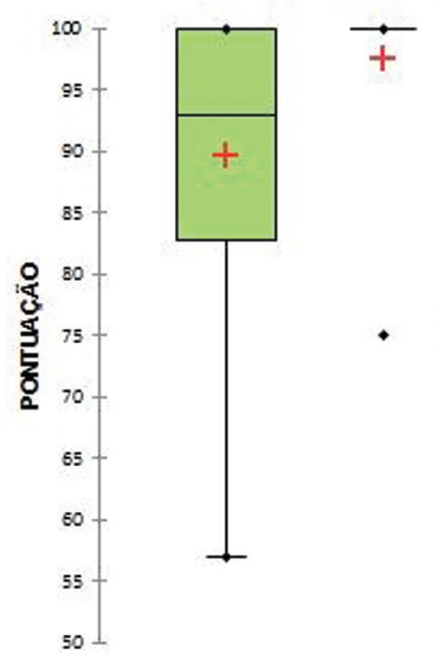

AOFAS MEDIOPÉ

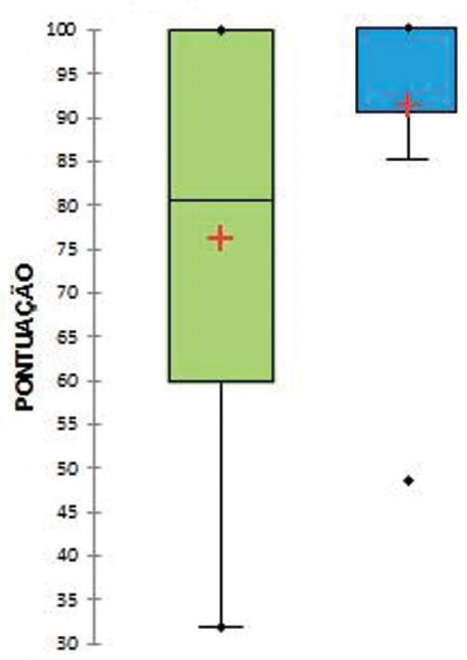

AOFAS RETROPÉ

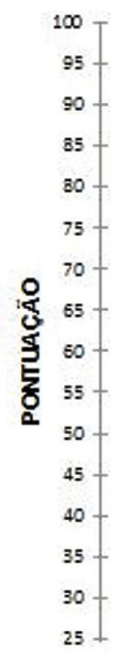

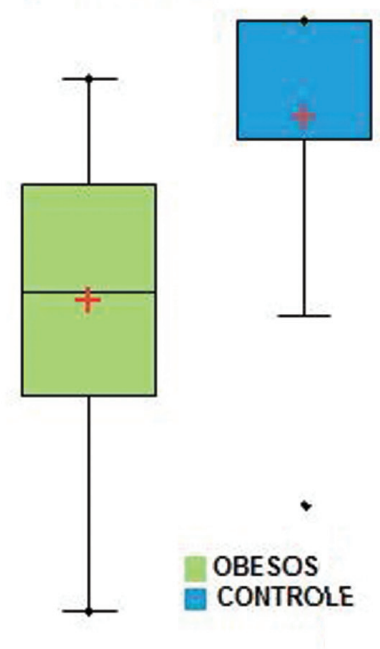

Fig. 2 Gráficos Box plot da escala funcional AOFAS das áreas do antepé, mediopé e retropé em indivíduos do grupo de estudo de obesos (à esquerda) e grupo controle, à direita.

Tabela 4 Resultados da correlação entre os subgrupos com IMC abaixo de 50 e acima de 50

\begin{tabular}{|l|l|l|l|}
\hline PARÂMETRO & OBESO IMC $<\mathbf{5 0}$ & OBESO IMC $>\mathbf{5 0}$ & ESTATÍSTICA \\
\hline \multirow{2}{*}{ EVA } & 91,6 & $100 \%$ & \multirow{2}{*}{$p=0,075$} \\
\cline { 2 - 3 } & Dor grave $=\mathbf{5 8 \%}$ & Dor grave $=31.2 \%$ & \\
\hline AOFAS Retropé & 65,62 & 66,25 & $p=0,428$ \\
\hline AOFAS Mediopé & 74,58 & 78,37 & $p=0,264$ \\
\hline
\end{tabular}

Abreviações: AOFAS, Associação Americana de Cirurgia do Pé e Tornozelo; EVA, escala visual analógica; IMC, índice de massa corporal.

Não houve diferenças estatísticas entre os grupos de obesos e controle em relação aos demais parâmetros avaliados (-Tabela 3 ).

A comparação entre os subgrupos de obesos mórbidos e supermórbidos não mostrou diferenças estatísticas em relação aos parâmetros avaliados (-Tabela 4).

\section{Discussão}

A casuística está de acordo com outros estudos na literatura ${ }^{2,11}$ que evidenciam uma prevalência aumentada de dor nos pés em indivíduos obesos em relação à população em geral. Neste trabalho, houve $95 \%$ de dor no grupo obeso e $40 \%$ no controle. Melo et al. ${ }^{12}$ descreveram dados similares com $85 \%$ de dor em membros inferiores em obesos mórbidos submetidos à gastroplastia redutora. Na população em geral, estes índices variam de $14 \%$ em adolescentes até $42 \%$ em populações $>65$ anos. ${ }^{11}$ No presente trabalho, ficou demonstrado OR de 4,2 de um indivíduo obeso grave (IMC $>40$ ) apresentar dor significativa no pé em relação a grupo controle de indivíduos com IMC médio de 24. Butterworh et al., ${ }^{2}$ em estudo de metanálise, descreveram OR de 3.1 de prevalência de dor nos pés de obesos em relação a pessoas com IMC $<25$.

A escala funcional AOFAS ${ }^{10}$ se mostrou mais alterada em seus três domínios nos indivíduos obesos da série em relação aos controles. Tal achado está de acordo com maior acometimento álgico referido na região posterior do calcâneo em obesos, ${ }^{2}$ bem como pela maior sobrecarga mecânica no mediopé que determinaria dor neste local. ${ }^{6,13}$ Os valores de OR em relação a grupo controle correspondentes ao retropé e mediopé nos indivíduos obesos graves (IMC $>40$ ), respectivamente de 4,81 e 3,33, ratificam a maior incidência de mau desempenho funcional para as atividades diárias, qualidade de vida e de deslocamento.

Embora a relação entre o peso e IMC aumentados e o surgimento de dor nos membros inferiores, à primeira vista, pareça se dever apenas a fatores biomecânicos determinados pela carga aumentada, há novas evidências relacionando a dor em articulações à síndrome metabólica sistêmica. ${ }^{2,14-16}$

$\mathrm{O}$ tecido adiposo visceral, bem como o tecido gorduroso claro depositado no tronco, se constituem em verdadeiros órgãos endócrinos que secretam citocinas, interleucinas, adipocinas e leptinas. ${ }^{17}$ A leptina estaria relacionada a efeitos pró-inflamatórios e à destruição de condrócitos. ${ }^{14}$

No presente estudo, não houve relação estatística entre a dor referida (EVA) e IMC ao se avaliar os componentes do grupo de obesos entre si. Outros autores ${ }^{2,11}$ também citaram que 0 IMC não estaria associado de forma independente com a prevalência de dor no pé. Butterworth et al. $^{2}$ ressaltaram que principalmente a massa de gordura elevada estaria relacionada a dor nos pés. A massa corpórea isoladamente não seria fator independente para dor. ${ }^{2,12}$ Estas observações sugerem a presença de fatores sistêmicos e não apenas biomecânicos determinando a gênese da dor no pé de indivíduos obesos. 
Há casuísticas ${ }^{18,19}$ na literatura que descreveram maior prevalência de pé plano em indivíduos obesos. No presente artigo, houve uma maior prevalência de indivíduos obesos com âng talocalcaneano aumentado, condizentes com pé plano. Os demais ângulos estudados não mostraram diferenças estatísticas em relação ao grupo controle. Alguns autores ${ }^{20}$ descreveram haver pouca relação entre dor em uma articulação e alterações radiográficas. Outro fator a se considerar é que indivíduos com obesidade mórbida tendem a ser mais jovens. ${ }^{21}$ Em virtude disto, ainda não teriam tido tempo para desenvolver artrose radiológica ou deformidades secundárias, por isso não seriam identificadas alterações à radiografia apesar da presença de dor. ${ }^{21}$

A atual pesquisa não mostrou diferença estatística nas medidas angulares obtidas nas radiografias para avaliar prevalência de alterações como hálux valgo (HV). A deformidade em HV não necessariamente estaria associada à dor. No entanto, a prevalência de HV em obesos tem mostrado resultados conflitantes na literatura. Frey et al. ${ }^{22}$ descreveram que HV estaria relacionado a IMC dentro da normalidade e não à obesidade. No entanto Cho et al..$^{23}$ relacionaram HV a índices aumentados de IMC. Já Nguyen et al. ${ }^{24}$ relataram que obesidade e sexo feminino seriam fatores protetores contra HV. Isto se daria pelo fato de mulheres com IMC $<25$ utilizarem com mais frequência sapatos com salto alto e bico fino enquanto obesos, por apresentarem pés mais largos, utilizariam sapatos com salto baixo e sem comprimir o antepé. ${ }^{24}$

Menz et al. ${ }^{25}$ descreveram maior prevalência de dor nos pés em mulheres em relação a homens (25\% versus $19 \%$ ) na comunidade de Framinghan, MA, EUA, em uma população geral. No entanto, no presente trabalho, não foram demonstradas diferenças entre os sexos masculino e feminino na prevalência e intensidade de dor nos pés nos casos de obesidade grave.

Em nossa casuística, não se demonstraram diferenças nos parâmetros funcionais dos pés pela escala AOFAS e no nível de dor (EVA) ao se comparar os subgrupos de obesos mórbidos e supermórbidos. Nossos dados são discordantes de outros autores que mostraram maior acometimento funcional em indivíduos superobesos. ${ }^{7}$ A pesquisa deve continuar e acumular uma quantidade maior de indivíduos para que, talvez, diferenças sejam evidenciadas.

No presente trabalho, como ponto de ressalva, deve-se citar que por se tratar de um estudo transversal, embora possam ocorrer correlações entre os parâmetros estudados, não há possibilidade de se determinar relação causa-efeito. Portanto, não é possível afirmar com os testes estatísticos empregados que a obesidade excessiva seria a responsável pelo quadro de dor, baixo nível funcional ou se estes fatores teriam efeito de causa reverso, ou seja, determinariam a ocorrência da obesidade pelo sedentarismo. Outro fator que merece ressalva foi a não discriminação da prevalência de comorbidades clínicas como hipertensão, diabetes e outras doenças sistêmicas entre os grupos de obesos e controle, o que pode ter gerado viés na análise dos dados. Além disso, a casuística estudada foi relativamente pequena, o que limita o alcance das conclusões. No entanto, deve-se ressaltar que o grupo de estudo se constitui em um universo muito específico de indivíduos com obesidade extremamente grave (mórbida com IMC > 40) e com indicação de cirurgia bariátrica, enquanto a maior parte dos dados da literatura pesquisada ${ }^{2,12}$ se referem a indivíduos com sobrepeso ou obesidade leve.

Foram demonstrados maior prevalência de dor e de comprometimento funcional envolvendo o pé na população obesa estudada. A não relação direta entre valores aumentados de IMC no grupo de obesos graves e dor aferida pela escala EVA corrobora as suspeitas de que mecanismos sistêmicos e não apenas fatores biomecânicos seriam determinantes do quadro álgico e de comprometimento funcional. Estes achados reforçam a importância do aprofundamento do estudo do acometimento do sistema músculoesquelético de pessoas com obesidade grave.

O perfil dos dados obtidos será válido no entendimento das patologias do sistema músculoesquelético decorrentes da síndrome metabólica da obesidade, podendo ser útil na formulação de condutas de abordagem preventiva e terapêuticas neste subgrupo especial de indivíduos.

\section{Conclusões}

A obesidade grave $(\mathrm{IM}>40)$ está relacionada a maior prevalência de dor nos pés. Os indivíduos com obesidade grave apresentam pior escore funcional (AOFAS) nas regiões do antepé, mediopé e retropé

\section{Conflito de Interesses}

Os autores declaram não haver conflito de interesses.

\section{Referências}

1 Thomas D, Elliott EJ. Low glycaemic index, or low glycaemic load, diets for diabetes mellitus. Cochrane Database Syst Rev 2009;(01): CD006296

2 Butterworth PA, Landorf KB, Smith SE, Menz HB. The association between body mass index and musculoskeletal foot disorders: a systematic review. Obes Rev 2012;13(07):630-642

3 Harms S, Larson R, Sahmoun AE, Beal JR. Obesity increases the likelihood of total joint replacement surgery among younger adults. Int Orthop 2007;31(01):23-26

4 Jinks C, Jordan K, Croft P. Disabling knee pain-another consequence of obesity: results from a prospective cohort study. BMC Public Health 2006;6:258

5 Martins GC, Martins Filho LF, Raposo AH, Gamallo RB, Menegazzi Z, Abreu AV. Radiographic evaluation and pain symptomatology of the knee in severely obese individuals - controlled transversal study. Rev Bras Ortop 2018;53(06):740-746

6 Mickle KJ, Steele JR. Obese older adults suffer foot pain and footrelated functional limitation. Gait Posture 2015;42(04):442-447

7 Fabris SM, Faintuch J, Brienze SL, et al. Are knee and foot orthopedic problems more disabling in the superobese? Obes Surg 2013;23(02):201-204

8 Sowers M, Karvonen-Gutierrez CA, Palmieri-Smith R, Jacobson JA, Jiang Y, Ashton-Miller JA. Knee osteoarthritis in obese women with cardiometabolic clustering. Arthritis Rheum 2009;61(10): 1328-1336

9 Li H, George DM, Jaarsma RL, Mao X. Metabolic syndrome and components exacerbate osteoarthritis symptoms of pain, depression and reduced knee function. Ann Transl Med 2016;4(07):133

10 Kitaoka HB, Alexander IJ, Adelaar RS, Nunley JA, Myerson MS, Sanders M. Clinical rating systems for the ankle-hindfoot, midfoot, hallux, and lesser toes. Foot Ankle Int 1994;15(07):349-353 
11 Menz H. Two feet, or one person? Problems associated with statistical analysis of paired data on foot and ankle medicine. Foot 2004;14(01):2-5

12 Melo IT, São-Pedro M. Musculoskeletal pain in lower limbs in obese patients before and after bariatric surgery. Arq Bras Cir Dig 2012;25(01):29-32

13 Walsh TP, Butterworth PA, Urquhart DM, et al. Increase in body weight over a two-year period is associated with an increase in midfoot pressure and foot pain. J Foot Ankle Res 2017;10:31

14 Gandhi R, Takahashi M, Smith H, Rizek R, Mahomed NN. The synovial fluid adiponectin-leptin ratio predicts pain with knee osteoarthritis. Clin Rheumatol 2010;29(11):1223-1228

15 Kalichman L, Li L, Kobyliansky E. Prevalence, pattern and determinants of radiographic hand osteoarthritis in Turkmen community-based sample. Rheumatol Int 2009;29(10):1143-1149

16 de Mello AP, Martins GCDS, Heringer AR, et al. Back pain and sagittal spine alignment in obese patients eligible for bariatric surgery. Eur Spine J 2019;28(05):967-975

17 Dumond H, Presle N, Terlain B, et al. Evidence for a key role of leptin in osteoarthritis. Arthritis Rheum 2003;48(11):3118-3129

18 Abdel-Fattah MM, Hassanin MM, Felembane FA, Nassaane MT. Flat foot among Saudi Arabian army recruits: prevalence and risk factors. East Mediterr Health J 2006;12(1-2):211-217
19 Otsuka R, Yatsuya H, Miura Y, et al. [Association of flatfoot with pain, fatigue and obesity in Japanese over sixties]. Nippon Koshu Eisei Zasshi 2003;50(10):988-998

20 Kinds MB, Welsing PM, Vignon EP, et al. A systematic review of the association between radiographic and clinical osteoarthritis of hip and knee. Osteoarthritis Cartilage 2011;19(07):768-778

21 Frilander H, Viikari-Juntura E, Heliövaara M, Mutanen P, Mattila VM, Solovieva S. Obesity in early adulthood predicts knee pain and walking difficulties among men: A life course study. Eur J Pain 2016;20(08):1278-1287

22 Frey C, Zamora J. The effects of obesity on orthopaedic foot and ankle pathology. Foot Ankle Int 2007;28(09):996-999

23 Cho NH, Kim S, Kwon DJ, Kim HA. The prevalence of hallux valgus and its association with foot pain and function in a rural Korean community. J Bone Joint Surg Br 2009;91(04): 494-498

24 Nguyen US, Hillstrom HJ, Li W, et al. Factors associated with hallux valgus in a population-based study of older women and men: the MOBILIZE Boston Study. Osteoarthritis Cartilage 2010;18(01): 41-46

25 Menz HB, Dufour AB, Casey VA, et al. Foot pain and mobility limitations in older adults: the Framingham Foot Study. J Gerontol A Biol Sci Med Sci 2013;68(10):1281-1285 